Voix et Images

volxetimages

\title{
Bibliographie de Jovette Marchessault
}

\section{Claudine Potvin}

Volume 16, numéro 2 (47), hiver 1991

Jovette Marchessault

URI : https://id.erudit.org/iderudit/200900ar

DOI : https://doi.org/10.7202/200900ar

Aller au sommaire du numéro

Éditeur(s)

Université du Québec à Montréal

ISSN

0318-9201 (imprimé)

1705-933X (numérique)

Découvrir la revue

Citer ce document

Potvin, C. (1991). Bibliographie de Jovette Marchessault. Voix et Images, 16(2),

272-280. https://doi.org/10.7202/200900ar d'utilisation que vous pouvez consulter en ligne.

https://apropos.erudit.org/fr/usagers/politique-dutilisation/ 


\section{Bibliographie de Jovette Marchessault}

\section{par Claudine Potvin, Université de l'Alberta}

\section{GUVRES}

\section{I.1 Volumes}

Comme une enfant de la terre / 1. Le crachat solaire, Montréal, Leméac, 1975 (prix France-Québec, 1976); traduit par: Yvonne M. Klein, Like a Child of the Earth, Vancouver, Talon Books, 1988. Premier roman d'une trilogie.

La Mère des herbes, Montréal, Éditions Quinze, 1980 (préface de Gloria F. Orenstein); traduit par Yvonne M. Klein, Mother of the Grass, Vancouver, Talon Books, 1989. Deuxième roman de la trilogie.

Tryptique lesbien, Montréal, les Éditions de la Pleine Lune, 1980 (postface de Gloria F. Orenstein); traduit par Yvonne M. Klein, Lesbian Triptych, Toronto, The Women's Press, 1985 (introduction et bibliographie de Barbara Godard. Texte composé de trois monologues: "Chronique du moyen-âge québécois "; * Les vaches de nuit * ; * Les faiseuses d'anges »).

Le deuxième monologue a été présenté à Paris au Lieu dit et en Italie au début de janvier 1979. Sous la direction de Pol Pelletier, il a été joué au Théâtre du Nouveau Monde le 5 mars 1979 dans le cadre du spectacle Célébrations consacré à l'écriture des femmes, organisé par Jovette Marchessault et Nicole Brossard. La représentation de ce monologue a été reprise au Théâtre expérimental des femmes en novembre 1979. De plus, il a été présenté en anglais, Night Cows, au Salon des femmes de New York à l'automne 1979, à la Galerie $A$ Space de Toronto le $1^{\text {er }}$ décembre 1980 et lors du colloque sur * Les femmes et les mots » qui a eu lieu à Vancouver en juillet 1983.

Le troisième monologue, *Les faiseuses d'anges" fut présenté à Montréal, au café-théâtre l'Ex-tasse au mois d'avril 1983.

La Saga des poules mouillées, Montréal, Éditions de la Pleine Lune, 1980 (éléments de correspondance entre l'auteure et Gloria F. Orenstein); $2^{e}$ édition, Montréal, Leméac, 1989 (introduction de Jacques LarueLanglois). Traduit par Linda Gaboriau, Saga of the Wet Hens, Vancouver, Talon Books, 1983.

La Saga des poules mouillées a été créée à Montréal, au Théâtre du Nouveau Monde le 24 avril 1981; la première production de la pièce en anglais fut présentée au Tarragon Theatre de Toronto le 18 février 1982.

La terre est trop courte, Violette Leduc, Montréal, Éditions de la Pleine Lune, 1982 (introduction de Francine Pelletier). 
Pièce créée à Montréal, àu Théâtre expérimental des femmes le 5 novembre 1981. Une première lecture du texte dans la traduction anglaise de Suzanne de Lotbinière a eu lieu lors d'une coproduction du Centre d'essai des auteurs dramatiques, du Playwrights Workshop de Montréal et Factory Theatre Lab de Toronto.

Lettre de Californie, Montréal, Éditions Nouvelle Optique, 1982.

Alice \& Gertrudé, Natalie \& Renée et ce cher Ernest, Montréal, Éditions de la Pleine Lune, 1984.

Pièce créée à Montréal, à l'Atelier continu le 20 octobre 1984 par les productions Vermeilles.

Anaïs, dans la queue de la comète, Montréal, Éditions de la Pleine Lune, 1985.

Pièce créée à Montréal, au Théâtre de Quat' Sous en septembre 1985.

Demande de travail sur les nébuleuses, Montréal, Leméac, 1988 (introduction de Pierre Filion).

Pièce créée à Montréal, au Théâtre d'aujourd'hui le 2 novembre 1988.

Des cailloux blancs pour les forêts obscures, Montréal, Leméac, 1987; traduit par Yvonne M. Klein, White Pebbles in the Dark Forests, Vancouver, Talon Books, 1990. Troisième volet de la trilogie romanesque.

Le Voyage magnifique d'Emily Carr, Montréal, Leméac, 1991.

Pièce créée à Montréal au Théâtre d'aujourd'hui en octobre 1990.

\section{I.2 Articles de périodiques}

«Faits d'hiver „, la Nouvelle Barre du jour, 68-69, septembre 1978, p. 129133.

«Nous n'écrirons plús pour les fonds de tiroirs *, Châtelaine, vol. XX, n 1 , janvier 1978, p. 48-49, 66-71.

*Les vaches de nuit *, la Nouvelle Barre du jour, 75, février 1979, p. 83-92.

«Il m'est encore impossible de chanter, mais j'écris », Jeu, 16, 1er trimestre 1980, p. 207-210.

«Les faiseuses d'anges », la Nouvelle Barre du jour, 87, février 1980, p. 19-29.

*Ainsi qu'une pensée cyclonale „, le Devoir, 19 avril 1980, p. 25.

*Les Monstresses », la Nouvelle Barre du jour, 102, avril 1981, p. 7-12.

*L'humanité des femmes *, le Devoir, 28 novembre 1981, p. 31.

"Pourquoi j'écris », Québec français, 47, octobre 1982,p. 33.

* Erreur sur le fruit „, la Vie en rose, 10, mars 1983, p. 48-49.

* Alice et Gertrude et Natalie et Renée et ce cher Ernest *, la Nouvelle Barre du jour, 132, 3, novembre 1983, p. 19-34.

*Alice and Gertrude and Natalie and Renée and Dear Ernest (traduction de Basil Kingstone), Canadian Fiction Magazine, 57, 1983, p. 58-64.

«Ce qui n'est pas dit „, le Devoir, 9 novembre 1984, p. 8. (lettre adressée à M. Robert Lévesque en réponse à son article sur Alice \& Gertrude [...], «Un 
texte coupé de sa sève», le Devoir, 31 octobre 1984, p. 9; R. Lévesque ajoute un très bref commentaire à la fin de la lettre de J. Marchessault.)

*Le sang des autres est le plus beau théâtre *, Etudes littéraires, vol. XVIII, $\mathrm{n}^{\circ} 3$, hiver 1985, p. 223-226.

« Requiem pour un serpent à sonnettes *, la Vie en rase, 37, juillet-août 1986 , p. 29-31.

\section{II. ÉTUDES}

\section{II.1 Généralités}

[ANONYME], «Prix France-Québec. Vient de paraître „, Lettres québécoises, 3 , septembre 1976, p. 17.

-, "Prix France-Québec et Prix Belgique-Canada ", Lettres québécoises, 4, novembre 1976, p. 44.

BELL, Gay, «Power, Magic and Visibility. From Biting Satirical Diatribes to Warm Evocations of the Natural Earth, Québecoise Playwright Jovette Marchessault captures the Strenght of Lesbian Energy. An Interview », The Body Politic, 122, janvier 1986, p. 34-35, 37.

BURGOYNE, Lynda, *Théâtre et homosexualité féminine, un continent invisible ", Jeu, 54, mars 1990, p. 114-118.

GABORIAU, Linda, *Jovette Marchessault: A Luminous Wake in Space ", Canadian Theatre Review, 43, été 1985, p. 91-98.

GAUDET, Gérald, *La passion de l'impossible», dans Voix d'écrivains. Entretiens, Montréal, Québec/Amérique, 1985, p. 120-128.

GODARD, Barbara, *Bibliography *, dans Lesbian Triptych (traduction de Yvonne M. Klein), Toronto, The Women's Press, 1985, p. 97-100.

, *Flying Away with Language*, dans Lesbian Triptych (traduction de Yvonne M. Klein), Toronto, The Women's Press, 1985, p. 9-28.

LAUTREDOUX, Florence, * Littérature féminine, littérature féministe dans le Québec contemporain. Ruptures et continuités \#, Etudes canadiennes, 18 , juin 1985 , p. 45-49.

LE CLÉZIO, Marguerite, * Poétique et/ou politique: le théâtre de Jovette Marchessault», North Dakota Quarterly, vol. LII, n 3, été 1984, p. 100 106.

MAHEU, Renée, «Jovette Marchessault. France-Québec », le Devoir, 24 décembre 1976, p. 18.

MARTIN, Jean-Guy, «Jovette Marchessault», le Journal de Montréal, 15 novembre 1980 , p. 17.

ORENSTEIN, Gloria F., «Jovette Marchessault ou la Quête extatique de la nouvelle chamane féministe „, Bulletin de la Société des professeurs de français en Amérique, 1, automne 1979, p. 37-57; paru en anglais sous le titre suivant: «Jovette Marchessault: The Ecstatic Vision-Quest of the new Feminist Shamane ", dans Lady Unique Inclination of the Night, automne 1980, p. 42-53, et dans Gynocritics / La gynocritique. Feminist Approaches to Writing by Canadian and Quebecoise Women / Approches 
féministes à l'écriture des Canadiennes et des Québécoises, sous la direction de Barbara Godard, Toronto, ECW Press, 1987, p. 179-187.

_, « Préface ", la Mère des herbes, Montréal, Quinze, 1980.

OUELLETTE-MICHALSKA, Madeleine, «Jovette Marchessault: envers et contre tous ", Perspectives, vol. XXIII, n ${ }^{\circ}$ 12, 21 mars 1981, p. 22-25.

ROSENFELD, Marthe, *The Development of a Lesbian Sensibility in the Work of Jovette Marchessault ", dans Traditionalism, Nationalism and Feminism: Women Writers of Quebec, sous la direction de Paula Gilbert Lewis, Westport, CT, Greenwood, 1985, p. 228-239.

ROYER, Jean, «Jovette Marchessault: ce chaos brûlant de la mémoire ", le Devoir, 31 octobre 1981, p. 21, 40; repris dans Ecrivains contemporains. Entretiens 3: 1980-1983, Montréal, l'Hexagone, 1985, p. 204-210.

RUPRECHT, Alvina, «Le discours dramatique de la femme. Langage et identité chez Jovette Marchessault et Denise Boucher „, Québec Studies, 3, 1985, p. 169-183.

SMITH, Donald, «Jovette Marchessault: de la femme tellurique à la mythification sociale » (entretien), Lettres québécoises, 27, automne 1982, p. 52-58.

STANTON, Julie, «Jovette Marchessault ç'a été: Tu crées ou tu crèves * (entretien), Châtelaine, 22, juin 1981, p. 110-114, 116, 118, 120.

\section{II.2 Sur les œuvres}

\section{II.2.1 Alice \& Gertrude, Natalie \& Renée et ce cher Ernest}

BERNATCHEZ, Raymond, «Jovette Marchessault et les amours lesbiens [sic]; à l'Atelier continu ", la Presse, 20 octobre 1984, p. E-4.

BERTIN, Raymond, «La pièce à Jovette», Relations, 506, décembre 1984, p. 338-339.

BOURASSA, André, «Images d'Amérique », Lettres québécoises, 38, été 1985, p. 47-48.

CORRIVAULT, Martine R., « $\Pi$ n'y a théâtre que si l'on joue», le Soleil, 8 décembre 1984, p. D-16.

LÉVESQUE, Robert, «Un texte coupé de sa sève », le Devoir, 31 octobre 1984, p. 9.

- «Ce qui n'est pas dit dans ma critique ", le Devoir, 9 novembre 1984, p. 8 (réponse à une lettre de Jovette Marchessault qui précède le commentaire de R. Lévesque).

PAQUETTE, Marie-Louise, «Où est la passion?», Jeu, 34, $1^{\text {er }}$ trimestre 1985, p. 128-131.

PEDNEAULT, Hélène, « Jovette Marchessault à l'Étang-aux-Oies. Avec Alice et Gertrude, Natalie et Renée et ce cher Ernest », la Vie en rose, 20, octobre 1984, p. 54-55 (entrevue à propos de la pièce).

ROBERT, Lucie, «Dramaturgie. La passion du dialogue », Voix \& images, vol. $\mathrm{XI}, \mathrm{n}^{\circ} 1$, automne 1985, p. 144.

ROYER, Jean, «Jovette Marchessault: soyons légendaires", le Devoir, 20 octobre 1984, p. 25. 


\section{II.2.2 Anaïs, dans la queue de la comète}

ACKERMAN, Marianne, «A Loving Portrait of Anais Nin», The Gazette, 28 septembre 1985, p. D-3.

AltONZO, Anne-Marie, «CEuvre de chair *, la Vie en rose, février 1986, p. 52.

LEFEBVRE, Paul, «Michèle Magny dans la lumière d'Anaiss», le Devoir, 21 septembre 1985, p. 27.

LÉVESQUE, Robert, *Marchessault, ou le théâtre pris dans sa pédagogie; Andrée Lachapelle sublime ", le Devoir, 27 septembre 1985, p. 22.

MOSS, Jane, "Anaïs, dans la queue de la comète", The French Review, vol. LX, no 5, avril 1987, p. 728-729.

ROBERT, Lucie, *Dramaturgie. La littérature au théâtre », Voix \& images, 34, automne 1986, p. 158.

\section{II.2.3 Des cailloux blancs pour les forêts obscures}

BASILE, Jean, «Les femmes de Jovette Marchessault „, la Presse, 17 octobre 1987 , p. J-4.

BOIVIN, Jean-Roch, «Donner naissance à des choses grandes et imparfaites ", le Devoir, 31 octobre 1987, p. D-3.

CLOUTIER, Guy, «Des cailloux blancs pour les forêts obscures, de Jovette Marchessault. Une célébration de ces femmes oubliées", le Soleil, 26 septembre 1987, p. F-8.

MARCOTTE, Hélène, «Des cailloux blancs pour les forêts obscures. Jovette Marchessault », Nuit blanche, 30, décembre-janvier 1987-1988, p. 13-14.

MILOT, Louise, *Des cailloux blancs pour les forêts obscures. Un Homme parmi les femmes ", Lettres québécoises, 48, hiver 1987-1988, p. 18-20.

ROY, Pierrette, «J'écris pour sauver mon âme... c'est mon destin ", la Tribune, 17 octobre 1987, p. B-9.

ROYER, Jean, «Jovette Marchessault. Le roman de la réconciliation», le Devoir, 8 octobre 1987, p. 15.

\section{II.2.4 Comme une enfant de la terre /1. Le crachat solaire}

BASILE, Jean, «Le Crachat solaire de Jovette Marchessault», le Devoir, 18 octobre 1975, p. 14.

HERZ, Micheline, *A Quebecois and an Acadian Novel Compared: The Use of Myth in Jovette Marchessault's Comme une enfant de la terre and Antonine Maillet's Pélagie-la-Charrette *, dans Traditionalism, Nationalism and Feminism: Women Writers of Quebec, sous la direction de Paula Gilbert Lewis, Westport, CT, Greenwood, 1985, p. 173-183.

RIOUX, Eliette, *Comme une enfant de la terre», les Têtes de pioche, 5, septembre 1976, p. 8.

\section{II.2.5 Demande de travail sur les nébuleuses}

LAVOIE, Pierre, «Parutions récentes », Jeu, 49, automne 1988, p. 256.

LÉPINE, Stéphane, «Le théâtre qu'on joue», Lettres québécoises, 53 , printemps 1989, p. 41-44. 
PONTAUT, Alain, «Dans le ciel d'une maison d'Amérique ", le Devoir, $1^{\text {er }}$ novembre 1988, p. 11-12.

ROBERT, Lucie, *Constellation classique», Voix \& images, 41, hiver 1989, p. 354.

\section{II.2.6 Lettre de Californie}

BAYARD, Caroline, *Marie José Thériault, Yolande Villemaire, Jovette Marchessault», Lettres québécoises, 29, printemps 1983, p. $42-45$ (voir «Lettre de Californie: ou commencer à écrire l'histoire „, p. 45).

LEWIS, Jocelyne, «Marchessault (Jovette). Lettre de Californie ", Nos livres, XIII, décembre $1982, n^{\circ} 475$.

ROGERS, Claire-Lise, *Zeus et Minerve », Canadian Literature, 98, automne 1983, p. 69-70.

ROYER, Jean, «Lettre d'amour de Jovette Marchessault", le Devoir, 13 novembre 1982, p. 19.

SUTHERLAND, Ronald, *A Fine Hommage to Women through the Centuries. Quebec Books», The Globe and Mail, 20 novembre 1982, p. L-8.

YANACOPOULO, Andrée, *Lettre de Montréal „, Spirale, 32, mars 1983, p. 4.

\section{II.2.7 La Mène des herbes}

COSSETTE, Gilles, «Le roman II: La Mère des herbes de Jovette Marchessault », Lettres québécoises, 20, hiver 1980-1981, p. 18-20.

L'HÉRAULT, Pierre, «Jovette Marchessault. La Mère des herbes. Quinze Éd.n, Livres et Auteurs québécois, 1980, p. 50-51.

ORENSTEIN, Gloria, «Plant Mother: Mythic Vision », New Women's Times: Feminist Review, 12, septembre-octobre 1980, p. 8-9.

OUELLETTE-MICHALSKA, Madeleine, «Au bout de l'absurde », Châtelaine, vol. XXI, $n^{\circ} 5$, mai 1980 , p. 8.

_. «ovette Marchessault: après la chute» le Devoir, 9 février 1980, p. 27.

THÉORET, France, «Un livre impatient: Marchessault, la Mère des herbes ", Spirale, 10, juin 1980, p. 1, 4.

\section{II.2.8 La Saga des poules mouillées}

[ANONYME], wovette Marchessault s'impose dans tout et le fait avec succès », la Tribune, 13 juin 1981, p. E-5.

__ «La Saga des poules mouillées, Monique Mercure seule à Toronto'”, le Journal de-Montréal, 18 février 1982, p. 61.

BELL, Gay, «Where Nest the Wet Hens», Broadside: A Feminist Review, février 1982, p. 12-13 (entretien avec Michelle Rossignol et Jovette Marchessault).

BOURASSA, André, «Le théâtre qu'on publie. Poules d'eau et vaches de nuit w, Lettres québécoises, 23, automne 1981, p. 37-38.

CARON, Claire, *Charlotte Boisjoli pathétique dans la Saga des poules mouillées », le Journal de Montréal, $1^{\mathrm{er}}$ mai 1981, p. 42. 
*Jovette Marchessault: Il est urgent d'écrire des rôles pour les femmes *, le Journal de Montréal, 23 avril 1981, p. 72.

CORBEIL, Carol, «Dream Comes True for Marchessault», The Globe and Mail, 28 mai 1981, p. 19.

_ Feminist Version of Quebec Writers \#, The Globe and Mail, 30 mai 1981, p. E-5.

DASSYLVA, Martial, *Marchessault au TNM. Sauvée par Rossignol ", la Presse, $1^{\text {er }}$ mai 1981, p. A-10.

—, «ovette Marchessault. L'écriture à voix haute. La Saga des poules mouillées », la Presse, 25 avril 1981, p. C-1.

DIONNE, André, «Le théâtre qu'on joue ", Lettres québécoises, 23, automne 1981, p. 39-41.

DUMONT, Monique, «Une filiation d'écriture: la Saga des poules mouillées », Spirale, 20, juin 1981, p. 19.

FORSYTH, Louise H., *Women Reclaim their Culture in Quebec: A Saga of Night Cows and Wet Hens", Spirale: A Women's Art and Culture Quarterly, vol. I, $n^{\circ}$ 2, automne 1981, p. 12-13 (en français, p. 13, 15).

HARRIS, Norma, * Magic of Wet Hens Misdirected », The Globe and Mail, 20 février 1982, p. E-9.

HOGUE, Jacqueline, «La Saga des poules mouillées », Nos livres, 13, juinjuillet $1982, \mathrm{n}^{\circ} 285$.

LARUE-LANGLOIS, Jacques, *Impossible de passer sous silence la Saga des poules mouillées », le Devoir, 15 juin 1981, p. 7.

MAROIS, Thérèse, *Mythes féministes: la Saga des poules mouillées au TNM », Jeu, 20, $3^{e}$ trimestre 1981, p. 52-56.

PETERSON, Maureen, *Artist, Novelist Tries her Hand at Play Writing ", The Gazette, 25 avril 1981, p. 116.

SABBATH, Lawrence, * Saga a Brillant, Imaginative Spectacle », The Gazette, 30 avril 1981, p. 28.

STANTON, Julie, «La Saga de Jovette Marchessault „, Livres d'ici, 2 septembre 1981, p. 32.

TALBOT, Michelle, «Une saga... à deux voix», Dimanche-Matin, 12 avril 1981 , p. B-7.

V., C., «La Saga des poules mouillées. Jovette Marchessault», le Temps fou, 15 , juin-août 1981, p. 60.

\section{II.2.9 La terre est trop courte, Violette Leduc}

BÉGUIN, Louis-Paul, "Les écrivaines », le Devoir, 4 décembre 1981, p. 18.

BOURASSA, André, «Le théâtre qu'on publie. Quand les poètes deviennent personnages ", Lettres québécoises, 26, été 1982, p. 45-47.

CONLOGUE, Ray, * Flaws Don't Diminish Play's Impact; Theatre Review of The Edge of the World is Too Near, Violette Leduc „, The Globe and Mail, 17 mai 1986, p. D-4. 
DASSYLVA, Martial, «Les tourbillons Leduc, Guilbeault et Marchessault", la Presse, 8 novembre 1981, p. C-4.

HALE, Amanda, *Voices, Vices and Voyeur. Review of The Edge of the earth is Too near, Violette Leduc, prod. Nightwood Theatre", Broadside: A Feminist Review, juin 1986, p. 11.

LARUE-LANGLOIS, Jacques, «La terre est trop courté, Violette Leduc au TEF. Un pas de géant pour le théâtre d'ici », le Devoir, 13 novembre 1981 , p. 14.

MERCIER-CROFT, Esther, *Nouveautés-Théâtre *, Québec français, 47, octobre 1982, p. 11.

MICHAUD, Ginette, « Marchessault, Jovette. La terre est trop courte, Violette Leduc ", Jeu, 24, 1982, p. 124-125.

MIETKIEWICZ, Henry, *What's the Big Deal here?; Theatre Review of The Edge of the World is Too Near, Violette Leduc ", The Toronto Star, 20 mai 1986, p. B-5.

\section{II.2.10 Tryptique lesbien}

ANDERSEN, Marguerite, «Lesbian Triptych; Book Review», Canadian Literature, 112, printemps 1987, p. 128-129; paru auparavant dans Resources for Feminist Research/Documentation sur la recherche féministe, vol. XV, ${ }^{\circ} 4$, décembre-janvier 1987, p. 61.

ARTEAU, Marcel, «Tryptique lesbien ", le Temps fou, 12, décembre-janvierfévrier 1980-1981, p. 57, 59, 61, 63.

CHITTICK, Kathryn, «Putrefying Sore», Canadian Literature, 112, printemps 1987, p. 128-129.

CONSTANTIN, Louise, "Tryptique lesbien", Des luttes et des rires de femmes, vol. IV, $\mathrm{n}^{\circ}$ 5, juin-juillet-août 1981, p. 43-44.

DIONNE, André, *Le thêâtre qu'on joue ", Lettres québécoises, 25, printemps 1982 , p. 50 (compte rendu de les Faiseuses d'anges présenté au caféthéâtre $l^{\prime} E x$-tasse).

—_ «ovette Marchessault. Tryptique lesbien, Éditions de la Pleine Lune ", Livres et Auteurs québécois, 1980, p. 51-52.

HALE, Amanda, *Day Dreams and Night Cows. Review of Lesbian Triptych ", Broadside: A Feminist Review, mai 1985, p. 13.

HANCOCK, Geoff, «Quebec Feminists Challenge Tradition», The Toronto Star, 24 août 1985, p. B-7.

JENOFF, Marvyne, «Lesbian Triptych, by Jovette Marchessault, translated by Yvonne M. Klein ", Books in Canada, vol. XIV, $n^{\circ} 6$, août-septembre 1985, p. 25.

NOËL, Francine, «Plaidoyer pour mon image , Jeu, 16, $3^{e}$ trimestre 1980 , p. 54 (sur * Les vaches de nuit»).

OUELLETTE-MICHALSKA, Madeleine, «À la Pleine Lune. De la colère à la tendresse ", le Devoir, 28 juin 1980, p. 15.

- _ « Jovette Marchessault à New York », le Devoir, 13 octobre 1979, p. 27 (au sujet de son exposition et de la représentation de Night's Cows). 
ROYER, Jean, * Jovette Marchessault: “I faut se rendre visible...": célébration des femmes au TNM, le Devoir, 3 mars 1979, p. 19 (sur le spectacle Célébrations organisé avec Nicole Brossard et dans le cadre duquel on a présenté les Vaches de nuit).

TRUDEL, Clément, «Deux performances de femmes», le Devoir, 15 avril 1983, p. 15 (sur * Les faiseuses d'anges *).

\section{II.2.11 Le Voyage magnifique d'Emily Carr}

LAMONTAGNE, Gilles R., «Jovette Marchessault. Faire connaitre des femmes qui ont apporté quelque chose à l'humanité», la Presse, 22 septembre 1990 , p. D-4.

\section{DIVERS: FILM, SPECTACLES, EXPOSITIONS, RADIO- TÉLÉVISION}

Les Terribles Vivantes, film de Dorothy T. Henaut, ONF, 1986 (film sur Louky Bersianik, Nicole Brossard et Jovette Marchessault). Voir Francine Laurendeau, * Terribles vivantes et hollandais chasseurs \#, le Devoir, 25 octobre 1986, p. D-6; de Simone Suchet, un commentaire sur le film dans Séquences, 129, avril 1987, p. 60.

Women's Salon de New. York, exposition/spectacle: Huit Montréalaises à New York, janvier 1980. Catalogue de Gloria F. Orenstein.

ORENSTEIN, Gloria F., Jovette Marchessault au Salon des femmes, New York, Montréal, Radio-Canada, octobre 1979.

Émission Visage, Radio-Québec, avril 1983 (entrevue). Voir Pascal Perreault, "Un attachant "visage" à Radio-Québec ", le Journal de Montréal, 20 avril 1983, p. 68.

* Le Retour de la déesse. Avec Françoise d'Eaubonne, Leonora Carrington ét Jovette Marchessault \#, Montréal, Radio-Canada (télévision), printemps 1980.

Une vingtaine d'expositions à Montréal, à la maison de la Sauvegarde et à la galerie Shayne (voir Sabbath, Lawrence, «Jovette Marchessault ", The Gazette, 18 avril 1981, p. 90), à Joliette et au Musée du Québec à Québec, à Toronto (galerie Shaw Remington, galerie A Space), au Centre culturel canadien de Paris et de Bruxelles ainsi qu'au Salon des femmes à New York (voir Orenstein, Gloria F., *The Telluric Women of Jovette Marchessault. Review of the Telluric sculptures „, Fireweed, 5-6, hiverprintemps 1978-1980, p. 164-165). 